\title{
ON PRINCIPAL FREQUENCIES, VOLUME AND INRADIUS IN CONVEX SETS
}

\author{
LORENZO BRASCO AND DARIO MAZZOLENI
}

\begin{abstract}
We provide a sharp double-sided estimate for Poincaré-Sobolev constants on a convex set, in terms of its inradius and $N$-dimensional measure. Our results extend and unify previous works by Hersch and Protter (for the first eigenvalue) and of Makai, Pólya and Szegö (for the torsional rigidity), by means of a single proof.
\end{abstract}

\section{CONTENTS}

1. Introduction 1

1.1. Principal frequencies and volume $\quad 1$

1.2. Principal frequencies and inradius 2

1.3. Interpolating between inradius and volume 4

1.4. Plan of the paper 6

2. Preliminaries 6

2.1. Notation 6

2.2. Inradius 6

2.3. Poincaré-Sobolev constants 8

2.4. The Minkowski functional 9

3. Lower bound 10

4. Upper bound 14

5. Further estimates 17

Appendix A. Some technical results 20

References $\quad 22$

\section{INTRODUCTION}

1.1. Principal frequencies and volume. For every open and bounded set $\Omega \subset \mathbb{R}^{N}$, we start by considering two of the most studied shape functionals. Namely, its principal frequency (or first eigenvalue of the Dirichlet-Laplacian) $\lambda(\Omega)$, and its torsional rigidity $T(\Omega)$. These are defined by

$$
\lambda(\Omega)=\inf _{\varphi \in C_{0}^{\infty}(\Omega) \backslash\{0\}} \frac{\int_{\Omega}|\nabla \varphi|^{2} d x}{\int_{\Omega}|\varphi|^{2} d x} \text { and } T(\Omega)=\sup _{\varphi \in C_{0}^{\infty}(\Omega) \backslash\{0\}} \frac{\left(\int_{\Omega}|\varphi| d x\right)^{2}}{\int_{\Omega}|\nabla \varphi|^{2} d x} .
$$

1991 Mathematics Subject Classification. 35P15, 49J40, 47A75.

Key words and phrases. Convex sets, nonlinear eigenvalue problems, torsional rigidity, inradius. 
These two quantities are well-studied and find many applications in different problems. In this paper, we will consider more generally the following generalized principal frequencies

$$
\lambda_{2, q}(\Omega)=\inf _{\varphi \in C_{0}^{\infty}(\Omega) \backslash\{0\}} \frac{\int_{\Omega}|\nabla \varphi|^{2} d x}{\left(\int_{\Omega}|\varphi|^{q} d x\right)^{\frac{2}{q}}},
$$

where $1 \leq q<2^{*}$, the latter being the usual Sobolev embedding exponent (see $[1,5,10]$ for some studies on these quantities). Observe that the two functionals $\lambda$ and $T$ are just particular instances of this more general family of Poincaré-Sobolev constants, indeed with the previous notation

$$
\lambda(\Omega)=\lambda_{2,2}(\Omega) \quad \text { and } \quad T(\Omega)=\frac{1}{\lambda_{2,1}(\Omega)} .
$$

The explicit computation of these quantities for a generic set $\Omega$ is a difficult task. Hence, it is of great importance (and interesting in itself) to provide sharp estimates for these functionals in terms of simpler quantities, often of geometric flavor.

The most celebrated estimate of this type is the so-called Faber-Krahn inequality, which asserts that

$$
\lambda_{2, q}(\Omega) \geq\left(\lambda_{2, q}\left(B_{1}\right) \omega_{N}^{\frac{2}{N}+\frac{2-q}{q}}\right)|\Omega|^{-\frac{2}{N}-\frac{2-q}{q}} .
$$

Here $B_{1} \subset \mathbb{R}^{N}$ denotes a ball of unit radius and $\omega_{N}=\left|B_{1}\right|$. In other words, it is possible to bound from below $\lambda_{2, q}$, in terms of (a negative power of) the volume of the set. Moreover, the estimate (1.2) is sharp, since the lower bound is (uniquely) attained by balls, up to sets of zero capacity ${ }^{1}$. We recall that (1.2) follows in a standard way from the variational characterization of $\lambda_{2, q}$, by using Schwarz symmetrization and the so-called Pólya-Szegö principle, see [13, Section 2].

In passing, we observe that $\lambda_{2, q}(\Omega)$ is not comparable with $|\Omega|^{-2 / N-(2-q) / q}$, not even among convex sets. For example, for a "slab-type" sequence, i.e.

$$
\Omega_{L}=\left(-\frac{L}{2}, \frac{L}{2}\right)^{N-1} \times(0,1), \quad L>0,
$$

we have (see for example Lemma A.2 below)

$$
\lim _{L \rightarrow+\infty} \lambda_{2, q}\left(\Omega_{L}\right)\left|\Omega_{L}\right|^{\frac{2}{N}+\frac{2-q}{q}}=+\infty .
$$

1.2. Principal frequencies and inradius. In order to clarify the scope of the present paper, it is useful to recall at this point that the sharp lower bound (1.2) may be quite weak for some classes of sets. For example, in the case $2 \leq q<2^{*}$, for the "slab-type" sequence, we have ${ }^{2}$

$$
\lambda_{2, q}\left(\Omega_{L}\right) \geq \frac{1}{C} \quad \text { while } \quad \lim _{L \rightarrow+\infty}\left|\Omega_{L}\right|^{-\frac{2}{N}-\frac{2-q}{q}}=0 .
$$

This shows that for sets of this type, the lower bound (1.2) is not very useful.

\footnotetext{
${ }^{1}$ By capacity of an open set $\Omega \subset \mathbb{R}^{N}$, we mean the quantity

$$
\operatorname{cap}(\Omega)=\inf _{u \in C_{0}^{\infty}\left(\mathbb{R}^{N}\right)}\left\{\int_{\mathbb{R}^{N}}|\nabla u|^{2} d x+\int_{\mathbb{R}^{N}} u^{2} d x: u \geq 1 \text { on } \Omega\right\},
$$

see $[8$, Chapter 4] for more details.

${ }^{2}$ Observe that $-\frac{2}{N}-\frac{2-q}{q}<0$ as $2 \leq q<2^{*}$.
} 
In this case, a more robust and precise lower bound would be given in terms of the inradius $R_{\Omega}$ of a set $\Omega$, i.e. the radius of the largest open ball contained in $\Omega$. However, a caveat is needed here: such a kind of lower bound can hold true only under some suitable geometric restrictions on the sets. This is due to the fact that while a principal frequency $\lambda_{2, q}$ is not affected by removing points (and, more generally, sets with zero capacity), this operation can strongly modify $R_{\Omega}$ : think for example of removing the center from a ball.

One possibility is to work with open bounded and convex sets. By still sticking to the case $q \geq 2$ we have (see [6, Proposition 6.3])

$$
\lambda_{2, q}(\Omega) \geq \frac{C_{N, q}}{R_{\Omega}^{2+\frac{2-q}{q} N}} .
$$

As usual in this type of estimates, the power on the inradius is dictated by scale invariance. Here $C_{N, q}>0$ is a universal constant, possibly depending on $N$ and $q$.

The value of the sharp constant in (1.3) is not known (see [6, Open Problem 1]), except that for the particular case $q=2$. In this case, we know that

$$
\lambda(\Omega)>\left(\frac{\pi}{2 R_{\Omega}}\right)^{2} .
$$

We also notice that inequality in (1.4) is strict among bounded convex sets, but the estimate is sharp. Indeed, for the "slab-type" sequence $\Omega_{L}$ we have

$$
\lim _{L \rightarrow+\infty} R_{\Omega_{L}}^{2} \lambda\left(\Omega_{L}\right)=\left(\frac{\pi}{2}\right)^{2}
$$

Estimate (1.4) has been first proved in two dimensions by Hersch in [14], by means of what he called évaluation par défaut. The extension to higher dimensions is usually attributed to Protter, see [19]. For this reason, we will refer to (1.3) and (1.4) as Hersch-Protter inequality.

In order to complete the picture, we also recall that $\lambda_{2, q}$ is actually comparable with a power of the inradius. Indeed, by employing the monotonicity with respect to set inclusion of $\lambda_{2, q}$, we easily get

$$
\lambda_{2, q}(\Omega) \leq \frac{\lambda_{2, q}\left(B_{1}\right)}{R_{\Omega}^{2+\frac{2-q}{q} N}}
$$

This inequality is optimal, as balls (uniquely) attain the equality cases. Moreover, the convexity requirement can now be dropped.

For the moment, we just discussed the Hersch-Protter estimate for the case $q \geq 2$. The reason is simple: in the case $1 \leq q<2$ the situation is entirely different. Indeed, as observed in [6, Proposition 6.1], it is not possible to a have a Hersch-Protter estimate in this regime. By calling again the "slab-type" sequence $\Omega_{L}$ into play, for $1 \leq q<2$ we have

$$
\lim _{L \rightarrow+\infty} \lambda_{2, q}\left(\Omega_{L}\right)=0 \quad \text { and } \quad R_{\Omega_{L}}=\frac{1}{2}, \text { for } L>1 .
$$

Thus (1.3) can not hold in this regime.

On the other hand, (1.5) immediately extends to this case, as well. 
1.3. Interpolating between inradius and volume. The last observation was the starting point of the investigation pursued in the present paper. In other words, we look for suitable "surrogates" of the Hersch-Protter estimate (1.3), in the case $1 \leq q<2$.

In order to do this, we take again the example of the "slab-type" sequence $\Omega_{L}$ and analyze the asymptotic behavior of $\lambda_{2, q}\left(\Omega_{L}\right)$. Indeed, by Lemma A.2 below we have

$$
0<\lim _{L \rightarrow+\infty} \lambda_{2, q}\left(\Omega_{L}\right)\left|\Omega_{L}\right|^{\frac{2-q}{q}} .
$$

This suggests that a suitable Hersch-Protter estimate could hold among convex sets, provided a multiplicative correction term containing a power of the volume is taken into account. It turns out that this intuition is correct and for $1 \leq q<2$ we have

$$
\lambda_{2, q}(\Omega)|\Omega|^{\frac{2-q}{q}} \geq \frac{C}{R_{\Omega}^{2}} .
$$

The case $q=2$ coincides with the Hersch-Protter inequality, but curiously enough this estimate does not extend to the super-homogeneous case $2<q<2^{*}$. Moreover, the quantity $\lambda_{2, q}(\Omega)|\Omega|^{(2-q) / q}$ is actually equivalent to $R_{\Omega}^{-2}$.

More precisely, the main results of this note are the following ones, whose proofs are contained in Sections 3 and 4 below. We refer to Section 2 for the definition of $\pi_{2, q}$.

Theorem 1.1 (Lower bound). Let $1 \leq q \leq 2$, for every $\Omega \subset \mathbb{R}^{N}$ open bounded convex set, we have

$$
\lambda_{2, q}(\Omega)|\Omega|^{\frac{2-q}{q}}>\left(\frac{\pi_{2, q}}{2 R_{\Omega}}\right)^{2} .
$$

The inequality is strict, but the estimate is sharp.

On the other hand, for $2<q<2^{*}$ we have

$$
\inf \left\{R_{\Omega}^{2} \lambda_{2, q}(\Omega)|\Omega|^{\frac{2-q}{q}}: \Omega \subset \mathbb{R}^{N} \text { open bounded convex }\right\}=0 .
$$

Theorem 1.2 (Upper bound). Let $1 \leq q<2^{*}$, for every $\Omega \subset \mathbb{R}^{N}$ open bounded convex set, we have

$$
\lambda_{2, q}(\Omega)|\Omega|^{\frac{2-q}{q}} \leq \frac{\omega_{N}^{\frac{2-q}{q}} \lambda_{2, q}\left(B_{1}\right)}{R_{\Omega}^{2}} .
$$

The inequality is attained if and only if $\Omega$ is a ball. Moreover, if $2 \leq q<2^{*}$, the inequality holds among open and bounded sets without the convexity assumption.

Remark 1.3 (Previous results). Our results extend to the case $q>1$ some previous results known for the case $q=1$. Indeed, by recalling that

$$
\lambda_{2,1}(\Omega)=\frac{1}{T(\Omega)},
$$

the estimates (1.6) and (1.7) can be rewritten as the double-sided control on the torsional rigidity

$$
\left(\frac{T\left(B_{1}\right)}{\omega_{N}}\right)|\Omega| R_{\Omega}^{2} \leq T(\Omega)<\frac{1}{3}|\Omega| R_{\Omega}^{2} .
$$

In dimension $N=2$, the lower bound is due to ${ }^{3}$ Pólya and Szegő (see [18, equation (7), page 100]), while the upper bound has been proved by Makai (see [17, equation (3')]). Both results have

\footnotetext{
${ }^{3}$ Caveat for the reader: in the notation of both [18] and [17], we have $4 T(\Omega)=P(\Omega)$.
} 
been generalized in [9] to every dimension $N \geq 2$ (see also [11, Theorem 1.1]). Moreover, both of them are sharp, as the lower bound is (uniquely) attained by balls, while the upper bound is asymptotically attained by the "slab-type" sequence $\Omega_{L}$.

In any case, we point out that our identification of equality cases in (1.7) appears to be new, even for the torsional rigidity, i.e. for the case $q=1$.

A comment on our proofs is in order.

Remark 1.4 (Method of proof). Our proof of (1.6) is different from the one by Makai, dealing with the case $q=1$. The latter seems quite difficult to adapt to the case $1<q<2$. Rather, we adapt a PDE-based technique used by Kajikiya in [15], to give a different proof of the Hersch-Protter estimate (1.4). We show that this technique is flexible enough to be adapted to the case $1 \leq q<2$, without loss of sharpness. This permits to unify the results of Makai and Hersch \& Protter, by means of a single proof.

For (1.7) we use the very same method of proof given by Pólya and Szegö for the case $q=1$. This is based on a variant of the so called method of interior parallels. This consists in choosing a suitable test function in the variational formulation (1.1): it turns out that a function of the Minkowski functional of $\Omega$ does the job. While in [18] the explicit form of the extremal of the ball is used, here we show that the knowledge of this explicit form is irrelevant. All that is needed is just that there exists an extremal function for $\lambda_{2, q}\left(B_{1}\right)$ which is radial. We also pay particular attention to the identification of equality cases, which is a bit subtle.

We now comment on the convexity assumption.

Remark 1.5 (Convexity matters). When $1 \leq q \leq 2$, both inequality (1.6) and inequality (1.7) can not hold for general open sets.

For the first one, we use again that removing points affects the inradius, but not a generalized principal frequency. That is, by taking the sequence of bounded open sets

$$
\Omega_{n}=(-n, n)^{N} \backslash\left\{x=\left(x_{1}, \ldots, x_{N}\right) \in \mathbb{Z}^{N}:\left|x_{i}\right| \leq n-1 \text { for } i=1, \ldots, N\right\},
$$

by scaling and using that points have zero capacity, we get

$$
\lambda_{2, q}\left(\Omega_{n}\right)\left|\Omega_{n}\right|^{\frac{2-q}{q}}=\lambda_{2, q}\left((-n, n)^{N}\right)(2 n)^{N \frac{2-q}{q}}=\frac{2^{N \frac{2-q}{q}} \lambda_{2, q}\left((-1,1)^{N}\right)}{n^{2}} .
$$

This implies that

$$
\lim _{n \rightarrow \infty} \lambda_{2, q}\left(\Omega_{n}\right)\left|\Omega_{n}\right|^{\frac{2-q}{q}}=0
$$

while it is easily seen that $R_{\Omega_{n}}=\sqrt{N} / 2$.

As a counterexample to (1.6), one can consider a disjoint union of balls

$$
\Omega_{n}=\bigcup_{i=1}^{n} B_{r_{i}}\left(x_{i}\right),
$$

with the radius given by

$$
r_{i}=\sqrt[N]{\frac{1}{i}}, \quad i \geq 1
$$

and the centers of the balls chosen so that $B_{r_{i}}\left(x_{i}\right) \cap B_{r_{j}}\left(x_{j}\right)=\emptyset$ for all $i \neq j$. This choice guarantees that

$$
R_{\Omega_{n}}=r_{1}=1 \quad \text { and } \quad \lim _{n \rightarrow \infty}\left|\Omega_{n}\right|=+\infty
$$


while by [7, Example 5.2] we have

$$
\lim _{n \rightarrow \infty} \lambda_{2, q}\left(\Omega_{n}\right)>0
$$

We point out that this is no more a counterexample as soon as $q \geq 2$, as the exponent of the measure term $(2-q) / q$ becomes non-positive.

1.4. Plan of the paper. After the Introduction, in Section 2 we fix the notation, recall some known facts about the Poincaré-Sobolev constants and give some properties of the Minkowski functional of a convex set. Section 3 is devoted to the proof of Theorem 1.1, while in Section 4 we prove the upper bound of Theorem 1.2. Finally, in Section 5 we discuss the case of more general versions of our "mixed" estimate, in terms of different powers of volume and inradius.

Acknowledgments. The initial input for this research has been a question raised by Andrea Malchiodi during a talk of the first author. We wish to thank him. We also thank Vladimir Bobkov, for pointing out the paper [15] to our attention.

D. M. has been supported by the INdAM-GNAMPA 2019 project "Ottimizzazione spettrale non lineare". Part of this work has been done during a visit of L. B. to Brescia and a visit of D. M. to Ferrara. The hosting institutions and their facilities are gratefully acknowledged.

\section{Preliminaries}

2.1. Notation. For the whole paper, $N \geq 2$ is the dimension of the space and we denote by $2^{*}$ the critical Sobolev exponent, i.e.

$$
2^{*}=\left\{\begin{array}{cc}
\frac{2 N}{N-2}, & \text { if } N \geq 3, \\
+\infty, & \text { if } N=2 .
\end{array}\right.
$$

For an open set $\Omega \subset \mathbb{R}^{N}$, we denote by $|\Omega|$ its $N$-dimensional Lebesgue measure and use the standard notation for the balls:

$$
B_{R}\left(x_{0}\right)=\left\{x \in \mathbb{R}^{N}:\left|x-x_{0}\right|<R\right\}, \quad \omega_{N}=\left|B_{1}(0)\right| .
$$

We will omit the center when this will coincide with the origin. Whenever it is well-defined, we call $\nu_{\Omega}(x)$ the outer unit normal versor at a point $x \in \partial \Omega$.

2.2. Inradius. For an open bounded set $\Omega \subset \mathbb{R}^{N}$ with Lipschitz boundary, we define the distance function from the boundary

$$
d_{\Omega}(x)=\inf _{y \in \partial \Omega}|x-y|, \quad \text { for } x \in \Omega .
$$

We recall that this is a 1 -Lipschitz function. Moreover, if $\Omega$ is convex, then $d_{\Omega}$ is concave and thus it is a weakly superharmonic function. It is well-known that the inradius $R_{\Omega}$ of $\Omega$ (i.e. the radius of the largest ball included in $\Omega$ ) coincides with

$$
R_{\Omega}=\sup _{x \in \Omega} d_{\Omega}(x)
$$

We present now a property of convex sets related to the inradius, which we will use in the proof of the rigidity for the upper bound (1.7). Though it should be somehow classical, we did not find a precise reference, so we give a proof for completeness. 
Lemma 2.1. Let $\Omega \subset \mathbb{R}^{N}$ be an open bounded convex set. Let us suppose that for some $R>0$, $B_{R} \subset \Omega$, then we have

$$
R \leq\left\langle x, \nu_{\Omega}(x)\right\rangle, \quad \text { for } \mathcal{H}^{N-1}-\text { a. e. } x \in \partial \Omega .
$$

Moreover, if $\Omega$ is of class $C^{1}$ and we have

$$
R=\left\langle x, \nu_{\Omega}(x)\right\rangle, \quad \text { for every } x \in \partial \Omega,
$$

then it must hold $\Omega=B_{R}$.

Proof. We observe that for every $x \in \partial \Omega$, by convexity

$$
\overline{B_{R}} \subset \bar{\Omega} \subset\left\{y \in \mathbb{R}^{N}:\left\langle y-x, \nu_{\Omega}(x)\right\rangle \leq 0\right\} .
$$

In particular, by taking the point $y=R \nu_{\Omega}(x) \in \partial B_{R}$, we get

$$
R=\left\langle R \nu_{\Omega}(x), \nu_{\Omega}(x)\right\rangle \leq\left\langle x, \nu_{\Omega}(x)\right\rangle .
$$

This proves (2.1).

We now suppose that $\Omega$ is of class $C^{1}$ and assume that equality in (2.1) holds for every $x \in \partial \Omega$. We argue by contradiction and suppose that $\Omega \neq B_{R}$. We take $x_{0} \in \partial \Omega$ such that

$$
T:=\operatorname{dist}\left(x_{0}, \partial B_{R}\right)=\max _{y \in \partial \Omega} \operatorname{dist}\left(y, \partial B_{R}\right) .
$$

By the contradiction assumption, we have $T>0$. Up to a rigid movement, we can suppose that

$$
x_{0}=(0, \ldots, 0,-T-R),
$$

and find a certain $r_{0}>0$ such that

$$
\Gamma:=\partial \Omega \cap\left(\left[-r_{0}, r_{0}\right]^{N-1} \times\left[-T-R-r_{0},-T-R+r_{0}\right]\right),
$$

coincides with the graph of a $C^{1}$ convex function $\Phi:\left[-r_{0}, r_{0}\right]^{N-1} \rightarrow \mathbb{R}$, with

$$
\Phi(0, \ldots, 0)=-T-R<-R .
$$

Moreover, by maximality of $x_{0}$, we have

$$
\nabla \Phi(0, \ldots, 0)=0
$$

see Figure 1. Then, at the point $x_{0}=(0, \ldots, 0,-T-R)$ we have $\nu_{\Omega}\left(x_{0}\right)=(0, \ldots, 0,-1)$. Thus, by using that we have equality in $(2.1)$, we get

$$
R=\left\langle x_{0}, \nu_{\Omega}\left(x_{0}\right)\right\rangle=T+R .
$$

Since $T>0$, this gives the desired contradiction.

Remark 2.2 (The importance of being $C^{1}$ ). The $C^{1}$ assumption is crucial to get the condition (2.2). On the other hand, when $\Omega$ is convex but not $C^{1}$, then it is no more true that

$$
\text { " } R=\left\langle x, \nu_{\Omega}(x)\right\rangle, \quad \text { for } \mathcal{H}^{N-1} \text {-a. e. } x \in \partial \Omega^{\prime \prime} \quad \Longrightarrow \quad \Omega=B_{R} .
$$

In fact, there are lots of convex sets for which this identity holds true. For example, it is sufficient to take any convex polyhedron, such that each of its faces touches the ball $B_{R}$. Another example can be found by taking the cone obtained as the convex envelope of $B_{R}$ and a point $x_{0} \in \mathbb{R}^{N} \backslash B_{R}$, see Figure 2. 


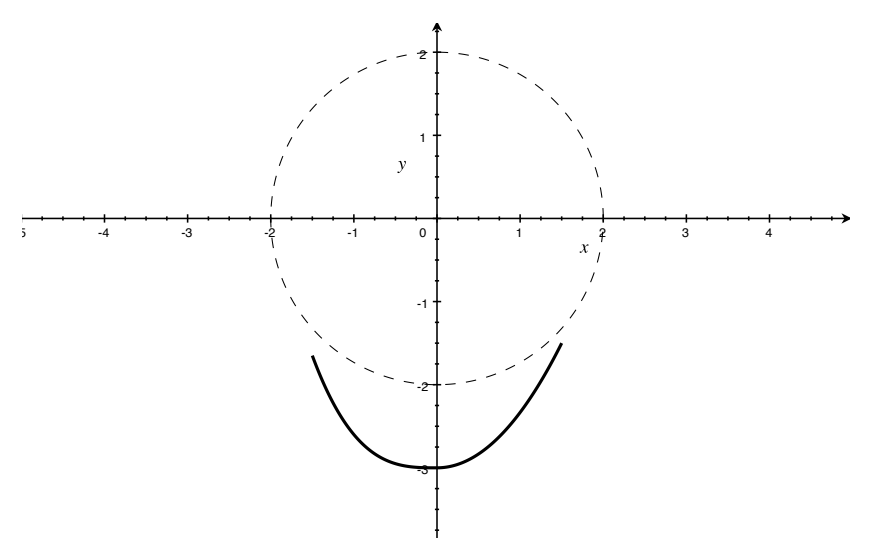

Figure 1. The geometric configuration in the proof of Lemma 2.1. The bold line represents the graph of $\Phi$.
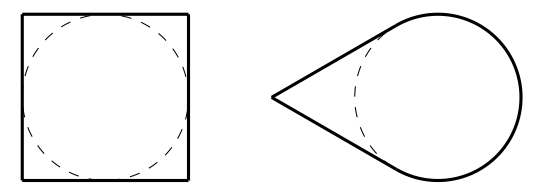

Figure 2. Two convex sets for which equality in (2.1) holds almost everywhere on the boundary.

2.3. Poincaré-Sobolev constants. For $1 \leq q<2^{*}$ and every open set $\Omega \subset \mathbb{R}^{N}$, we have defined in (1.1) the Poincaré-Sobolev constants, which we can interpret as generalizations of the first eigenvalue of the Dirichlet-Laplacian. They can be equivalently characterized as

$$
\lambda_{2, q}(\Omega)=\inf _{\varphi \in C_{0}^{\infty}(\Omega)}\left\{\int_{\Omega}|\nabla \varphi|^{2} d x:\|\varphi\|_{L^{q}(\Omega)}=1\right\} .
$$

If $\Omega$ is bounded or, more generally, has finite measure, the infimum is attained on the homogeneous Sobolev space $\mathcal{D}_{0}^{1,2}(\Omega)$, defined as the completion of $C_{0}^{\infty}(\Omega)$ with respect to the norm

$$
u \mapsto\|\nabla u\|_{L^{2}(\Omega)}
$$

In this case, a minimizer $u \in \mathcal{D}_{0}^{1,2}(\Omega)$ of the previous problem weakly solves the Lane-Emden equation

$$
-\Delta u=\lambda_{2, q}(\Omega)|u|^{q-2} u, \quad \text { in } \Omega .
$$


As we already recalled in the introduction, the quantity $1 / \lambda_{2,1}(\Omega)$ coincides with the torsional rigidity $T(\Omega)$.

From the definition, it is easy to check that these quantities scale as

$$
\lambda_{2, q}(t \Omega)=t^{-2-\frac{2-q}{q} N} \lambda_{2, q}(\Omega), \quad t>0 .
$$

Finally, as it is clear from the statement of the Theorem 1.1, the constants $\pi_{2, q}$ play a fundamental role in our work. These are nothing but the one-dimensional Poincaré-Sobolev constants, more precisely they are defined by

$$
\pi_{2, q}=\min _{\varphi \in W^{1,2}((0,1)) \backslash\{0\}}\left\{\frac{\left\|\varphi^{\prime}\right\|_{L^{2}((0,1))}}{\|\varphi\|_{L^{q}((0,1))}}: \varphi(0)=\varphi(1)=0\right\} .
$$

We refer to [5, Appendix A] and [12, Section 5] for more details. It is worth recalling some explicit values for these constants, see [5, Remark 2.4],

$$
\pi_{2,1}=2 \sqrt{3} \quad \text { and } \quad \pi_{2,2}=\pi .
$$

Then it is immediate to see that Makai's upper bound in (1.8) coincides the lower bound in (1.6) when $q=1$, while the Hersch-Protter estimate (1.4) is contained again in (1.6) when $q=2$.

The relation between the constants $\pi_{2, q}$ and $\lambda_{2, q}$ for the "slab-type" sequence $\Omega_{L}$ is detailed in the Appendix, see Lemma A.2.

2.4. The Minkowski functional. Here we recall the definition and main properties of the Minkowski functional of a convex set $\Omega \subset \mathbb{R}^{N}$ such that $0 \in \Omega$, denoted by $j_{\Omega}$. This is defined by

$$
j_{\Omega}(x):=\inf \{r>0: x \in r \Omega\} .
$$

First of all, by construction it is easily seen that

$$
\left\{x \in \mathbb{R}^{N}: j_{\Omega}(x)=t\right\}=t(\partial \Omega), \quad \text { for every } t>0 .
$$

The main properties of $j_{\Omega}$ needed for our purposes are summarized in the following

Lemma 2.3. Let $\Omega \subset \mathbb{R}^{N}$ be an open bounded convex set, such that $0 \in \Omega$. The function $j_{\Omega}$ is a convex Lipschitz and positively 1-homogeneous function, i.e.

$$
j_{\Omega}(t x)=t j_{\Omega}(x), \quad \text { for every } x \in \mathbb{R}^{N}, t>0 .
$$

Moreover, $j_{\Omega}$ is differentiable for $\mathcal{H}^{N-1}$-almost every $x \in \partial \Omega$ and it holds

$$
\left\langle x, \nu_{\Omega}(x)\right\rangle=\frac{1}{\left|\nabla j_{\Omega}(x)\right|}, \quad \text { for } \mathcal{H}^{N-1} \text {-a.e. } x \in \partial \Omega .
$$

Proof. For completeness, we sketch the proof of these classical facts. The homogeneity of $j_{\Omega}$ is a straightforward consequence of its definition. Moreover, by still using its definition, it is not difficult to see that $j_{\Omega}$ is level convex, i.e.

$$
j_{\Omega}((1-t) z+t w) \leq \max \left\{j_{\Omega}(z), j_{\Omega}(w)\right\}, \quad \text { for every } z, w \in \mathbb{R}^{N}, t \in[0,1] .
$$

By using this property with

$$
z=\frac{x}{j_{\Omega}(x)}, \quad w=\frac{y}{j_{\Omega}(y)}, \quad t=\frac{j_{\Omega}(y)}{j_{\Omega}(x)+j_{\Omega}(y)}
$$

and using the positive 1 -homogeneity, we then get that $j_{\Omega}$ is sub-additive. Finally, from this we obtain

$$
j_{\Omega}((1-t) x+t y) \leq j_{\Omega}((1-t) x)+j_{\Omega}(t y)=(1-t) j_{\Omega}(x)+t j_{\Omega}(y),
$$


i.e. $j_{\Omega}$ is convex.

As a convex function, it is automatically locally Lipschitz. By positive 1-homogeneity, we can upgrade this information to a global Lipschitz continuity. In any case, we have that $j_{\Omega}$ is differentiable almost everywhere in $\mathbb{R}^{N}$.

To prove that $j_{\Omega}$ is differentiable almost everywhere on $\partial \Omega$, we use again the positive 1-homogeneity: indeed, if there exists $\Sigma \subset \partial \Omega$ such that

$$
\mathcal{H}^{N-1}(\Sigma)>0
$$

and $j_{\Omega}$ is not differentiable on $\Sigma$, then $j_{\Omega}$ would automatically be not differentiable on the cone generated by $\Sigma$, i.e.

$$
C_{\Sigma}=\{x \in \Omega: x=t y \text { for some } t \in[0,1], y \in \Sigma\} .
$$

But this would be a set with positive $N$-dimensional measure, on which $j_{\Omega}$ is not differentiable, thus giving a contradiction.

Finally, by differentiating in $t$ the identity

$$
j_{\Omega}(t x)=t j_{\Omega}(x),
$$

and taking $t=1$, we get

$$
\left\langle\nabla j_{\Omega}(x), x\right\rangle=j_{\Omega}(x) .
$$

By recalling (2.3), the latter implies

$$
\left|\nabla j_{\Omega}(x)\right|\left\langle\nu_{\Omega}(x), x\right\rangle=1, \quad \text { for } \mathcal{H}^{N-1} \text {-a. e. } x \in \partial \Omega .
$$

This concludes the proof.

\section{LOWER BOUND}

In order to prove Theorem 1.1, the following technical result will be useful. The proof is standard, we give it for completeness.

Lemma 3.1. Let $q \geq 1$ and $f \in C^{2}([a, b])$ be a non-decreasing function, such that

$$
-f^{\prime \prime}=C f^{q-1}, \quad \text { in }[a, b], \quad \text { with } f(a)=0 .
$$

Let $\Omega \subset \mathbb{R}^{N}$ be an open set and let $u \in W^{1,2}(\Omega) \cap L^{\infty}(\Omega)$ be a weakly superhamonic function, i.e.

$$
\int_{\Omega}\langle\nabla u, \nabla \varphi\rangle d x \geq 0, \quad \text { for every } \varphi \in C_{0}^{\infty}(\Omega) \text { with } \varphi \geq 0 .
$$

Let us assume in addition that

$$
a \leq u(x) \leq b, \quad \text { for a. e. } x \in \Omega .
$$

Then the composition $\phi=f \circ u$ satisfies $-\Delta \phi \geq C \phi^{q-1}|\nabla u|^{2}$ in weak sense, i.e.

$$
\int_{\Omega}\langle\nabla \phi, \nabla \varphi\rangle d x \geq C \int_{\Omega} \phi^{q-1}|\nabla u|^{2} \varphi d x
$$

for every $\varphi \in C_{0}^{\infty}(\Omega)$ with $\varphi \geq 0$.

Proof. We take $\eta \in C_{0}^{\infty}(\Omega)$ non-negative and insert in (3.1) the test function ${ }^{4}$

$$
\varphi=f^{\prime}(u) \eta \geq 0 \text {. }
$$

\footnotetext{
${ }^{4}$ Observe that this $\varphi$ is only a $W^{1,2}$ function with compact support in $\Omega$, but by a standard density argument it is clearly admissible.
} 
We obtain

$$
\begin{aligned}
0 \leq \int_{\Omega}\left\langle\nabla u, \nabla\left(f^{\prime}(u) \eta\right)\right\rangle d x & =\int_{\Omega}|\nabla u|^{2} f^{\prime \prime}(u) \eta d x \\
& +\int_{\Omega}\left\langle f^{\prime}(u) \nabla u, \nabla \eta\right\rangle d x \\
& =-C \int_{\Omega}|\nabla u|^{2} f(u)^{q-1} \eta d x \\
& +\int_{\Omega}\langle\nabla f(u), \nabla \eta\rangle d x
\end{aligned}
$$

By recalling the definition of $\phi=f \circ u$, this gives the desired result.

We are now in position to prove Theorem 1.1.

Proof of Theorem 1.1. We divide the proof in three parts.

1. Inequality for $1 \leq q \leq 2$. We adapt the trick of [15] for proving the Hersch-Protter inequality (1.4), i. e. for the case $q=2$. We take $v \in W^{1,2}((-1,0))$ to be a positive solution of

$$
\min _{\varphi \in W^{1,2}((-1,0))}\left\{\int_{-1}^{0}\left|\varphi^{\prime}\right|^{2} d t: \int_{-1}^{0}|\varphi|^{q} d t=1 \text { and } \varphi(-1)=0\right\}
$$

We can assume that $v$ is non-decreasing: indeed, if this were not the case, we could consider the new function

$$
w(t)=\int_{-1}^{t}\left|v^{\prime}(\tau)\right| d \tau
$$

which is positive and non-decreasing by construction and such that $w(-1)=0$. Moreover, we have

$$
\int_{-1}^{0}\left|w^{\prime}(t)\right|^{2} d t=\int_{-1}^{0}\left|v^{\prime}(t)\right|^{2} d t
$$

and

so that

$$
w(t)^{q}=\left(\int_{-1}^{t}\left|v^{\prime}(\tau)\right| d \tau\right)^{q} \geq\left|\int_{-1}^{t} v^{\prime}(\tau) d \tau\right|^{q}=|v(t)|^{q}
$$

$$
\int_{-1}^{0} w^{q} d t \geq 1
$$

This implies that $\widetilde{w}=w\|w\|_{L^{q}((-1,0))}^{-1}$ is another positive minimizer of (3.2), and we can work with it.

By recalling that the minimal value (3.2) coincides with $\left(\pi_{2, q} / 2\right)^{2}$ (see [5, Lemma A.1]), we get that $v$ is a positive and non-decreasing function that solves the following mixed problem

$$
\left\{\begin{aligned}
-v^{\prime \prime} & =\left(\frac{\pi_{2, q}}{2}\right)^{2} v^{q-1}, \quad \text { in }(-1,0) \\
v(-1) & =v^{\prime}(0)=0
\end{aligned}\right.
$$

Actually, by using the equation it is easily seen that $v$ is of class $C^{2}$ on the interval $[-1,0]$. We then consider the function (recall the notation of Subsection 2.2)

$$
\phi(x)=v\left(\frac{d_{\Omega}(x)}{R_{\Omega}}-1\right),
$$


and observe that

$$
\left|\nabla d_{\Omega}\right|^{2}=1, \quad \text { a. e. in } \Omega
$$

and that $d_{\Omega}$ is weakly superharmonic in $\Omega$, thanks to the convexity of the set. We can then apply Lemma 3.1 with the choices

$$
f=v \quad \text { and } \quad u=\frac{d_{\Omega}}{R_{\Omega}}-1,
$$

and obtain that

$$
\left(\frac{\pi_{2, q}}{2 R_{\Omega}}\right)^{2} \int_{\Omega} \phi^{q-1} \varphi d x \leq \int_{\Omega}\langle\nabla \phi, \nabla \varphi\rangle d x, \quad \text { for every } \varphi \in C_{0}^{\infty}(\Omega), \varphi \geq 0 .
$$

By a standard density argument, in the previous equation we can also admit test functions in $W^{1,2}$ with compact support in $\Omega$. In particular, by taking $\varphi=\eta^{2} / \phi$ with $\eta \in C_{0}^{\infty}(\Omega)$ and using Picone's inequality, we get

$$
\left(\frac{\pi_{2, q}}{2 R_{\Omega}}\right)^{2} \int_{\Omega} \phi^{q-2} \eta^{2} d x \leq \int_{\Omega}\left\langle\nabla \phi, \nabla\left(\frac{\eta^{2}}{\phi}\right)\right\rangle d x \leq \int_{\Omega}|\nabla \eta|^{2} d x .
$$

This in particular implies that

$$
\left(\frac{\pi_{2, q}}{2 R_{\Omega}}\right)^{2} \leq \frac{\int_{\Omega}|\nabla \eta|^{2} d x}{\int_{\Omega} \phi^{q-2} \eta^{2} d x} .
$$

We now observe that by Hölder's inequality we have

$$
\int_{\Omega} \phi^{q-2} \eta^{2} d x \geq \frac{\left(\int_{\Omega} \eta^{q} d x\right)^{\frac{2}{q}}}{\left(\int_{\Omega} \phi^{q} d x\right)^{\frac{2-q}{q}}} .
$$

By using this in (3.4) and then taking the infimum over $\eta$, we obtain

$$
\left(\frac{\pi_{2, q}}{2 R_{\Omega}}\right)^{2} \leq \lambda_{2, q}(\Omega)\left(\int_{\Omega} \phi^{q} d x\right)^{\frac{2-q}{q}} .
$$

If $q=2$ the proof is over and we obtain the Hersch-Protter estimate. If $1 \leq q<2$, we are now left with estimating from above the $L^{q}$ norm of $\phi$. By using the definition of $\phi$, the Coarea Formula and property (3.3), we get

$$
\int_{\Omega} \phi^{q} d x=\int_{0}^{R_{\Omega}}\left(v\left(\frac{t}{R_{\Omega}}-1\right)\right)^{q} P\left(\Omega_{t}\right) d t,
$$

where we set

$$
\Omega_{t}=\left\{x \in \Omega: d_{\Omega}(x)>t\right\},
$$

and by $P\left(\Omega_{t}\right)$ we denote the perimeter of the set $\Omega_{t}$. We recall that the perimeter is monotone increasing with respect to set inclusion, in the class of convex sets (see [8, Lemma 2.2.2]). Since $\Omega_{t}$ is convex, we get that the function $\psi(t)=P\left(\Omega_{t}\right)$ is monotone decreasing. Moreover, if we define

$$
\xi(t)=\int_{0}^{t}\left(v\left(\frac{\tau}{R_{\Omega}}-1\right)\right)^{q} d \tau, \quad \text { for } t \in\left[0, R_{\Omega}\right],
$$


then it is easily seen that the pair $(\xi, \psi)$ verifies the assumptions of Lemma A.1. Indeed, by using that $v$ is monotone non-decreasing on $[-1,0]$, we can infer that

$$
\xi(t) \leq t\left(v\left(\frac{t}{R_{\Omega}}-1\right)\right)^{q}=t \xi^{\prime}(t),
$$

which entails that $t \mapsto \xi(t) / t$ is increasing. By applying Lemma A.1, we thus get

$$
\begin{aligned}
\int_{\Omega} \phi^{q} d x & =\int_{0}^{R_{\Omega}}\left(v\left(\frac{t}{R_{\Omega}}-1\right)\right)^{q} P\left(\Omega_{t}\right) d t \\
& \leq \frac{\xi\left(R_{\Omega}\right)}{R_{\Omega}} \int_{0}^{R_{\Omega}} P\left(\Omega_{t}\right) d t=\frac{\xi\left(R_{\Omega}\right)}{R_{\Omega}}|\Omega| .
\end{aligned}
$$

It is only left to observe that by definition of $\xi$, with a simple change of variable we have

$$
\frac{\xi\left(R_{\Omega}\right)}{R_{\Omega}}=\frac{1}{R_{\Omega}} \int_{0}^{R_{\Omega}}\left(v\left(\frac{\tau}{R_{\Omega}}-1\right)\right)^{q} d \tau=\int_{-1}^{0} v^{q} d s=1,
$$

where we have used that the function $v$ has unit $L^{q}$ norm on the interval $[-1,0]$. This shows that

$$
\int_{\Omega} \phi^{q} d x \leq|\Omega|
$$

Finally, by spending this information into (3.5), we get the desired estimate.

2. Sharpness for $1 \leq q \leq 2$. To prove the sharpness we consider the "slab-type" sequence

$$
\Omega_{L}=\left(-\frac{L}{2}, \frac{L}{2}\right)^{N-1} \times(0,1) .
$$

By Lemma A.2 below, we know that

$$
\lambda_{2, q}\left(\Omega_{L}\right) \sim \frac{\left(\pi_{2, q}\right)^{2}}{L^{(N-1) \frac{2-q}{q}}}, \quad \text { as } L \rightarrow+\infty .
$$

On the other hand, by construction it is easy to check that

$$
R_{\Omega_{L}}=\frac{1}{2} \quad \text { and } \quad\left|\Omega_{L}\right| \sim L^{N-1}, \quad \text { as } L \rightarrow+\infty .
$$

Finally, we obtain

$$
\lambda_{2, q}\left(\Omega_{L}\right)\left|\Omega_{L}\right|^{\frac{2-q}{q}} \sim\left(\frac{\pi_{2, q}}{2 R_{\Omega_{L}}}\right)^{2}, \quad \text { as } L \rightarrow+\infty,
$$

so we have proved the sharpness of the estimate (1.6).

3. The case $2<q<2^{*}$. We still take the family of sets $\Omega_{L}$ as above. In this case, by Lemma A.2 we have

$$
\lambda_{2, q}\left(\Omega_{L}\right) \sim \lambda_{2, q}\left(\mathbb{R}^{N-1} \times(0,1)\right)>0, \quad \text { as } L \rightarrow+\infty .
$$

Thus, we now get

$$
R_{\Omega_{L}}^{2} \lambda_{2, q}\left(\Omega_{L}\right)\left|\Omega_{L}\right|^{\frac{2-q}{q}} \sim \frac{1}{4} \lambda_{2, q}\left(\mathbb{R}^{N-1} \times(0,1)\right) L^{(N-1) \frac{2-q}{q}}, \quad \text { as } L \rightarrow+\infty,
$$

and this quantity converges to 0 , thanks to the fact that $2-q<0$. This concludes the proof. 


\section{UPPER BOUND}

The proof of the upper bound is based on the use of a clever test function. The idea is quite similar to the so-called method of interior parallels. The latter uses test functions of the form

$$
u(x)=\varphi\left(d_{\Omega}(x)\right) .
$$

In our case, on the contrary, test functions of the form

$$
u(x)=\varphi\left(j_{\Omega}(x)\right),
$$

will do the job. As recalled in Section $2, j_{\Omega}$ is the Minkowski functional of a convex set $\Omega \subset \mathbb{R}^{N}$ such that $0 \in \Omega$. Its relevant properties needed in the following proof are contained in Lemma 2.3.

Proof of Theorem 1.2. We divide the proof in two cases, depending on whether $q<2$ or $q \geq 2$.

Case $2 \leq q<2^{*}$. In this case, the proof is trivial. It is sufficient to observe that, for all $\Omega \subset \mathbb{R}^{N}$ open and bounded, we have

$$
R_{\Omega}^{2} \lambda_{2, q}(\Omega)|\Omega|^{\frac{2-q}{q}}=\left(\lambda_{2, q}(\Omega) R_{\Omega}^{2-\frac{2-q}{q} N}\right)\left(\frac{|\Omega|}{R_{\Omega}^{N}}\right)^{\frac{2-q}{q}},
$$

and use that both quantities are (uniquely) maximized by balls among open sets. In fact, by monotonicity of $\lambda_{2, q}$ with respect to set inclusion, we have

$$
\lambda_{2, q}(\Omega) \leq \lambda_{2, q}\left(B_{R_{\Omega}}\right)=\lambda_{2, q}\left(B_{1}\right) R_{\Omega}^{2-\frac{2-q}{q} N} .
$$

On the other hand, since $\Omega$ contains a ball of volume $\omega_{N} R_{\Omega}^{N}$, it is clear that

$$
\frac{R_{\Omega}^{N}}{|\Omega|} \leq \frac{1}{\omega_{N}}
$$

By recalling that $2-q \leq 0$, we get the conclusion.

Case $1 \leq q<2$. By definition of inradius, we have that $\Omega$ contains a ball of radius $R_{\Omega}$. Without loss of generality, we can assume that such a ball is centered at the origin.

We take $u \in \mathcal{D}_{0}^{1,2}\left(B_{1}\right)$ to be optimal for the variational problem defining $\lambda_{2, q}\left(B_{1}\right)$. Without loss of generality, we can take $u$ to be positive. Moreover, we know that it must be a radially symmetric function (see [16, Theorem 3]). Thus, there exists a $C^{1}$ function $f:[0,1] \rightarrow[0,+\infty)$ such that

$$
u(x)=f(|x|), \quad \text { for every } x \in B_{1} .
$$

The previous properties of $u$ entail that $f$ is decreasing and that $f^{\prime}(0)=0$. By using spherical coordinates, we have

$$
\lambda_{2, q}\left(B_{1}\right)=\frac{N \omega_{N} \int_{0}^{1}\left|f^{\prime}(t)\right|^{2} t^{N-1} d t}{\left(N \omega_{N} \int_{0}^{1} f(t)^{q} t^{N-1} d t\right)^{\frac{2}{q}}} .
$$

We then use the composition $f \circ j_{\Omega}$ as a test function in the Rayleigh quotient defining $\lambda_{2, q}(\Omega)$. Indeed, $f$ is $C^{1}, j_{\Omega}$ is Lipschitz and observe that we have

$$
f\left(j_{\Omega}(x)\right)=f(1)=0, \quad \text { for every } x \in \partial \Omega,
$$


so that $f \circ j_{\Omega} \in \mathcal{D}_{0}^{1,2}(\Omega)$. We first compute the $L^{q}$ norm of this test function. By using the Coarea Formula and the property (2.3), we have

$$
\int_{\Omega} f\left(j_{\Omega}\right)^{q} d x=\int_{0}^{1} f(t)^{q}\left(\int_{t \partial \Omega} \frac{1}{\left|\nabla j_{\Omega}(x)\right|} d \mathcal{H}^{N-1}\right) d t .
$$

By using the change of variable $x=t y$ and the fact that $\nabla j_{\Omega}$ is positively 0 -homogeneous, we get

$$
\int_{\Omega} f\left(j_{\Omega}\right)^{q} d x=\left(\int_{0}^{1} f(t)^{q} t^{N-1} d t\right)\left(\int_{\partial \Omega} \frac{1}{\left|\nabla j_{\Omega}(x)\right|} d \mathcal{H}^{N-1}\right) .
$$

If we further use (2.4) and the Divergence Theorem, we finally get

$$
\begin{aligned}
\int_{\Omega} f\left(j_{\Omega}\right)^{q} d x & =\left(\int_{0}^{1} f(t)^{q} t^{N-1} d t\right)\left(\int_{\partial \Omega}\left\langle x, \nu_{\Omega}\right\rangle d \mathcal{H}^{N-1}\right) \\
& =N|\Omega| \int_{0}^{1} f(t)^{q} t^{N-1} d t .
\end{aligned}
$$

We proceed similarly, in order to estimate the Dirichlet integral. By using again the Coarea Formula, (2.3) and the change of variable $x=t y$ as above, we have

$$
\int_{\Omega}\left|\nabla f\left(j_{\Omega}\right)\right|^{2} d x=\left(\int_{0}^{1}\left|f^{\prime}(t)\right|^{2} t^{N-1} d t\right)\left(\int_{\partial \Omega}\left|\nabla j_{\Omega}\right| d \mathcal{H}^{N-1}\right) .
$$

We use again (2.4), so to obtain

$$
\int_{\Omega}\left|\nabla f\left(j_{\Omega}\right)\right|^{2} d x=\left(\int_{0}^{1}\left|f^{\prime}(t)\right|^{2} t^{N-1} d t\right)\left(\int_{\partial \Omega} \frac{1}{\left\langle x, \nu_{\Omega}\right\rangle} d \mathcal{H}^{N-1}\right) .
$$

We now estimate the scalar product. By Lemma 2.1, we have

$$
R_{\Omega} \leq\left\langle x, \nu_{\Omega}(x)\right\rangle, \quad \text { for } \mathcal{H}^{N-1} \text {-a. e. } x \in \partial \Omega .
$$

Thus we get the following lower bound

$$
\left\langle x, \nu_{\Omega}\right\rangle=\frac{\left\langle x, \nu_{\Omega}\right\rangle^{2}}{\left\langle x, \nu_{\Omega}\right\rangle} \geq \frac{R_{\Omega}^{2}}{\left\langle x, \nu_{\Omega}\right\rangle}, \quad \text { for } \mathcal{H}^{N-1}-\text { a. e. } x \in \partial \Omega .
$$

By inserting this into (4.3), we get

$$
\begin{aligned}
\int_{\Omega}\left|\nabla f\left(j_{\Omega}\right)\right|^{2} d x & \leq \frac{1}{R_{\Omega}^{2}}\left(\int_{0}^{1}\left|f^{\prime}(t)\right|^{2} t^{N-1} d t\right)\left(\int_{\partial \Omega}\left\langle x, \nu_{\Omega}\right\rangle d \mathcal{H}^{N-1}\right) \\
& =\frac{N|\Omega|}{R_{\Omega}^{2}}\left(\int_{0}^{1}\left|f^{\prime}(t)\right|^{2} t^{N-1} d t\right) .
\end{aligned}
$$


Now, by putting together (4.5) and (4.2), we obtain

$$
\begin{aligned}
\lambda_{2, q}(\Omega) & \leq \frac{\int_{\Omega}\left|\nabla f\left(j_{\Omega}\right)\right|^{2} d x}{\left(\int_{\Omega} f\left(j_{\Omega}\right)^{q} d x\right)^{\frac{2}{q}}} \\
& \leq \frac{\omega_{N}^{\frac{2}{q}-1}|\Omega|^{1-\frac{2}{q}}}{R_{\Omega}^{2}} \frac{\left(N \omega_{N} \int_{0}^{1}\left|f^{\prime}(t)\right|^{2} t^{N-1} d t\right)}{\left(N \omega_{N} \int_{0}^{1} f(t)^{q} t^{N-1} d t\right)^{\frac{2}{q}}} \\
& =\frac{\omega_{N}^{\frac{2}{q}-1}|\Omega|^{1-\frac{2}{q}}}{R_{\Omega}^{2}} \lambda_{2, q}\left(B_{1}\right),
\end{aligned}
$$

where in the last equality we used (4.1). By rearranging the terms, it is immediate to see that we have proved the claimed inequality (1.7).

As for the equality cases, it is easy to see that if equality holds in (1.7) for an open bounded convex set $\Omega$ containing the origin, then all the inequalities in (4.6) must become equalities and in particular we deduce that

$$
f \circ j_{\Omega} \text { is optimal for } \lambda_{2, q}(\Omega)
$$

and

$$
R_{\Omega}=\left\langle x, \nu_{\Omega}(x)\right\rangle, \quad \text { for } \mathcal{H}^{N-1}-\text { a. e. } x \in \partial \Omega .
$$

By optimality, the first condition (4.7) implies that $f \circ j_{\Omega}$ is a weak solution of

$$
-\Delta u=C u^{q-1}, \quad \text { in } \Omega .
$$

In particular, by Elliptic Regularity this implies that $f \circ j_{\Omega}$ is locally smooth in $\Omega$, say $C^{1}$. By writing

$$
j_{\Omega}(x)=f^{-1} \circ\left(f \circ j_{\Omega}\right)(x), \quad \text { for } x \in \Omega,
$$

and observing that $f^{-1}$ is $C^{1}$ except at zero, we get that

$$
j_{\Omega} \in C^{1}(\Omega \backslash\{0\}) .
$$

This in turn implies that a set $\Omega$ attaining the equality in (1.7) is necessarily of class $C^{1}$. By using the second information (4.8) and the identification of equality cases in Lemma 2.1, we finally obtain that $\Omega$ must be a ball.

Remark 4.1. In [4] the following scale invariant quantity has been studied

$$
\frac{\lambda(\Omega) T(\Omega)}{|\Omega|},
$$

among the class of open bounded convex sets. In [4, Theorem 1.4] the authors give the following lower bounds

and

$$
\frac{\lambda(\Omega) T(\Omega)}{|\Omega|} \geq\left(\frac{\pi}{2}\right)^{2} \frac{1}{N^{N+2}(N+2)}, \quad \text { for } N \geq 3,
$$

$$
\frac{\lambda(\Omega) T(\Omega)}{|\Omega|} \geq\left(\frac{\pi}{2}\right)^{2} \frac{1}{12}, \quad \text { for } N=2 .
$$


By recalling that in our notation

$$
\lambda(\Omega)=\lambda_{2,2}(\Omega) \quad \text { and } \quad T(\Omega)=\frac{1}{\lambda_{2,1}(\Omega)},
$$

we can rewrite the previous functional as

$$
\frac{\lambda(\Omega) T(\Omega)}{|\Omega|}=\frac{\lambda_{2,2}(\Omega) R_{\Omega}^{2}}{R_{\Omega}^{2} \lambda_{2,1}(\Omega)|\Omega|} .
$$

If we now use the Hersch-Protter inequality to estimate the numerator from below and Theorem 1.2 with $q=1$ to estimate the denominator from above, we get

$$
\frac{\lambda(\Omega) T(\Omega)}{|\Omega|}=\frac{\lambda_{2,2}(\Omega) R_{\Omega}^{2}}{R_{\Omega}^{2} \lambda_{2,1}(\Omega)|\Omega|} \geq\left(\frac{\pi}{2}\right)^{2} \frac{1}{\lambda_{2,1}\left(B_{1}\right) \omega_{N}} .
$$

By further using that

we end up with

$$
\frac{1}{\lambda_{2,1}\left(B_{1}\right) \omega_{N}}=\frac{T\left(B_{1}\right)}{\omega_{N}}=\frac{1}{N(N+2)}
$$

$$
\frac{\lambda(\Omega) T(\Omega)}{|\Omega|} \geq\left(\frac{\pi}{2}\right)^{2} \frac{1}{N(N+2)},
$$

which improves strictly the lower bound of [4, Theorem 1.4], in every dimension $N \geq 2$. We refer to [3, Theorems $1.4 \& 1.5]$ for a finer lower bound in a restricted class of convex planar sets, as well as to [2, Conjecture 4.2] for the conjectured sharp lower bound.

\section{Further Estimates}

Our main results can be seen as a double-sided sharp estimate on the shape functional

$$
\Omega \mapsto R_{\Omega}^{2} \lambda_{2, q}(\Omega)|\Omega|^{\frac{2-q}{q}},
$$

in the class of open and bounded convex sets. In a natural way, one could ask whether a similar result can be obtained for the more general shape functional

$$
\Omega \mapsto R_{\Omega}^{\beta} \lambda_{2, q}(\Omega)|\Omega|^{\alpha},
$$

where $\alpha, \beta \in \mathbb{R}$ are two arbitary exponents.

Of course, the two exponents $\alpha, \beta$ must satisfy some restrictions. The first one is that of scale invariance. This imposes that we must have

$$
\beta=2-N\left(\alpha-\frac{2-q}{q}\right) .
$$

Then we set

$$
\mathfrak{m}(\alpha):=\inf \left\{R_{\Omega}^{2-N\left(\alpha-\frac{2-q}{q}\right)} \lambda_{2, q}(\Omega)|\Omega|^{\alpha}: \Omega \subset \mathbb{R}^{N} \text { open bounded convex }\right\},
$$

and

$$
\mathfrak{M}(\alpha)=\sup \left\{R_{\Omega}^{2-N\left(\alpha-\frac{2-q}{q}\right)} \lambda_{2, q}(\Omega)|\Omega|^{\alpha}: \Omega \subset \mathbb{R}^{N} \text { open bounded convex }\right\} .
$$

Observe that our Theorems 1.1 and 1.2 correspond to $\alpha=(2-q) / q$. For the quantity (5.1) we have the following 
Proposition 5.1 (Minimization). Let $1 \leq q<2^{*}$ and let $\alpha \in \mathbb{R}$ be an exponent. We have

$$
\mathfrak{m}(\alpha)>0 \quad \Longleftrightarrow \quad \alpha \geq \max \left\{\frac{2-q}{q}, 0\right\} .
$$

Moreover, if ${ }^{5}$

$$
\alpha \geq \frac{2-q}{q}+\frac{2}{N}
$$

balls uniquely minimize (5.1), even when the convexity assumption is dropped.

Proof. Let us assume that $\mathfrak{m}(\alpha)>0$. We consider the usual family of sets

$$
\Omega_{L}=\left(-\frac{L}{2}, \frac{L}{2}\right)^{N-1} \times(0,1)
$$

and observe that, taking into account Lemma A.2, we have the following.

If $1 \leq q \leq 2$

$$
R_{\Omega_{L}}^{2-N\left(\alpha-\frac{2-q}{q}\right)} \lambda_{2, q}\left(\Omega_{L}\right)\left|\Omega_{L}\right|^{\alpha} \sim C_{N, q, \alpha} \frac{\left(\pi_{2, q}\right)^{2}}{L^{(N-1) \frac{2-q}{q}}} L^{(N-1) \alpha},
$$

as $L \rightarrow+\infty$.

If $2<q<2^{*}$

$$
R_{\Omega_{L}}^{2-N\left(\alpha-\frac{2-q}{q}\right)} \lambda_{2, q}\left(\Omega_{L}\right)\left|\Omega_{L}\right|^{\alpha} \sim C_{N, q, \alpha} \lambda_{2, q}\left(\mathbb{R}^{N-1} \times(0,1)\right) L^{(N-1) \alpha},
$$

as $L \rightarrow+\infty$.

Thus the assumption $\mathfrak{m}(\alpha)>0$ entails that we must have

$$
\alpha \geq \frac{2-q}{q}, \quad \text { if } 1 \leq q \leq 2, \quad \text { and } \quad \alpha \geq 0, \quad \text { if } 2<q<2^{*},
$$

as desired.

Let us now assume that

$$
\alpha \geq \max \left\{\frac{2-q}{q}, 0\right\} .
$$

If $1 \leq q \leq 2$, we can rewrite our functional as follows

$$
R_{\Omega}^{2-N\left(\alpha-\frac{2-q}{q}\right)} \lambda_{2, q}(\Omega)|\Omega|^{\alpha}=\left(R_{\Omega}^{2} \lambda_{2, q}(\Omega)|\Omega|^{\frac{2-q}{q}}\right)\left(\frac{|\Omega|}{R_{\Omega}^{N}}\right)^{\alpha-\frac{2-q}{q}},
$$

and observe that both terms are bounded from below by a positive constant, in the class of convex sets. For the first one, it is sufficient to use our estimate (1.6), while for the second one we can use that

$$
\frac{|\Omega|}{R_{\Omega}^{N}} \geq \omega_{N}
$$

\footnotetext{
${ }^{5}$ Observe that
}

$$
\frac{2-q}{q}+\frac{2}{N}>0
$$

thanks to the fact that $1 \leq q<2^{*}$. 


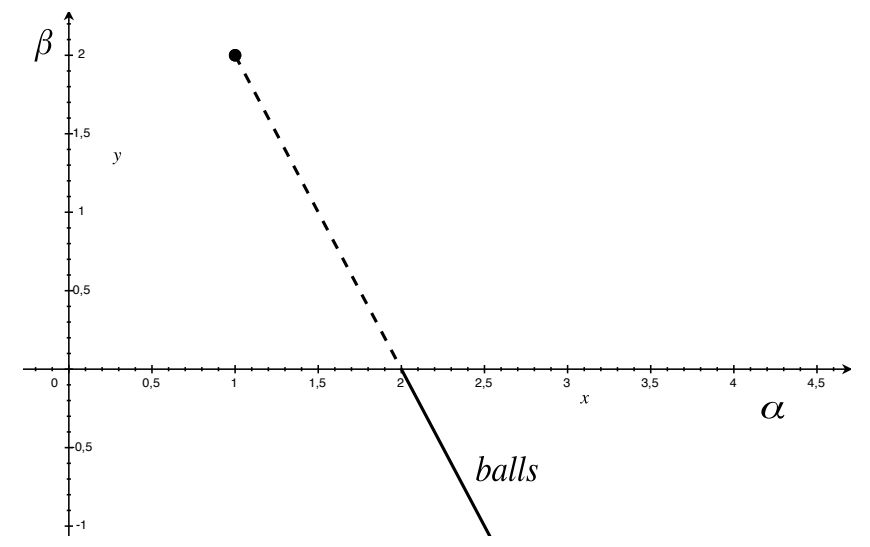

FIGURE 3. A diagram illustrating the result of Lemma 5.1, for the minimization of the quantity

$$
\Omega \mapsto R_{\Omega}^{\beta} \lambda_{2, q}(\Omega)|\Omega|^{\alpha} .
$$

For simplicity we draw it for the case $N=2$ and $q=1$. The black dot corresponds to the case of our Main Theorem, i.e. $\alpha=(2-q) / 2=1$ and $\beta=2$. In this case, slab-type sequences give the optimal lower bound (5.1). The continuous black line corresponds to the cases where balls are extremals. The dotted line corresponds to the open cases, where the infimum $\mathfrak{m}(\alpha)$ is non-trivial, but its value is not known.

If $2<q<2^{*}$, we can instead rewrite our functional as follows

$$
R_{\Omega}^{2-N\left(\alpha-\frac{2-q}{q}\right)} \lambda_{2, q}(\Omega)|\Omega|^{\alpha}=\left(R_{\Omega}^{2-\frac{2-q}{q} N} \lambda_{2, q}(\Omega)\right)\left(\frac{|\Omega|}{R_{\Omega}^{N}}\right)^{\alpha},
$$

and use that for a open convex set $\Omega \subset \mathbb{R}^{N}$ we have (see [6, Proposition 6.3])

$$
R_{\Omega}^{2-\frac{2-q}{q} N} \lambda_{2, q}(\Omega) \geq C_{N, q},
$$

and again (5.3). In both cases, we get $\mathfrak{m}(\alpha)>0$.

Finally, in the case

$$
\alpha \geq \frac{2-q}{q}+\frac{2}{N},
$$

it is enough to rewrite the functional in the following form

$$
R_{\Omega}^{2-N\left(\alpha-\frac{2-q}{q}\right)} \lambda_{2, q}(\Omega)|\Omega|^{\alpha}=\left(\lambda_{2, q}(\Omega)|\Omega|^{\frac{2}{N}+\frac{2-q}{q}}\right)\left(\frac{|\Omega|}{R_{\Omega}^{N}}\right)^{\alpha-\left(\frac{2-q}{q}+\frac{2}{N}\right)},
$$

and notice that both quantities are (uniquely) minimized by balls. The first one thanks to the Faber-Krahn inequality and the second one again by (5.3). The proof is concluded.

For the quantity (5.2) the situation is simpler and the picture is complete.

Proposition 5.2 (Maximization). Let $1 \leq q<2^{*}$ and let $\alpha \in \mathbb{R}$ be an exponent. We have

$$
\mathfrak{M}(\alpha)<+\infty \quad \Longleftrightarrow \quad \alpha \leq \max \left\{\frac{2-q}{q}, 0\right\} .
$$


Moreover, in this case balls uniquely attains $\mathfrak{M}(\alpha)$.

Proof. Let us assume that $\mathfrak{M}(\alpha)<+\infty$. The asserted restriction on $\alpha$ can then be obtained as before, by considering the family of sets

$$
\Omega_{L}=\left(-\frac{L}{2}, \frac{L}{2}\right)^{N-1} \times(0,1),
$$

we leave the details to the reader.

We now assume that

$$
\alpha \leq \max \left\{\frac{2-q}{q}, 0\right\} .
$$

For $1 \leq q \leq 2$, we rewrite our functional as follows

$$
R_{\Omega}^{2-N\left(\alpha-\frac{2-q}{q}\right)} \lambda_{2, q}(\Omega)|\Omega|^{\alpha}=\left(R_{\Omega}^{2} \lambda_{2, q}(\Omega)|\Omega|^{\frac{2-q}{q}}\right)\left(\frac{|\Omega|}{R_{\Omega}^{N}}\right)^{\alpha-\frac{2-q}{q}},
$$

and observe that both terms are (uniquely) maximized by balls. The first one thanks to our estimate (1.7), the second one by (5.3) (observe that the exponent $\alpha-(2-q) / q$ is non-positive).

For $2<q<2^{*}$, our assumption on $\alpha$ entails that $\alpha \leq 0$ and we use instead the following rewriting

$$
R_{\Omega}^{2-N\left(\alpha-\frac{2-q}{q}\right)} \lambda_{2, q}(\Omega)|\Omega|^{\alpha}=\left(R_{\Omega}^{2-\frac{2-q}{q} N} \lambda_{2, q}(\Omega)\right)\left(\frac{|\Omega|}{R_{\Omega}^{N}}\right)^{\alpha} .
$$

Here as well, both terms are (uniquely) maximized by balls. For the first one, it is sufficient to use the monotonicity of $\lambda_{2, q}$ with respect to set inclusion, while for the second one we use (5.3), as usual.

\section{Appendix A. Some technical Results}

The following simple one-dimensional result was an essential ingredient for the proof of the lower bound (1.6).

Lemma A.1. Let $a>0$ and let $\xi:[0, a] \rightarrow \mathbb{R}$ be an absolutely continuous function such that

$$
\xi(0)=0 \quad \text { and } \quad t \mapsto \frac{\xi(t)}{t} \text { is non-decreasing. }
$$

Let $\psi:[0, a] \rightarrow[0,+\infty)$ be a non-increasing function. Then we have

$$
\int_{0}^{a} \xi^{\prime}(t) \psi(t) d t \leq \frac{\xi(a)}{a} \int_{0}^{a} \psi(t) d t .
$$

Proof. Without loss of generality we can suppose that $\psi$ is smooth. By integrating by parts and observing that $\xi(0)=0$, we have

$$
\begin{aligned}
\int_{0}^{a} \xi^{\prime}(t) \psi(t) d t & =\xi(a) \psi(a)+\int_{0}^{a} \xi(t)\left(-\psi^{\prime}(t)\right) d t \\
& =\xi(a) \psi(a)+\int_{0}^{a} \frac{\xi(t)}{t} t\left(-\psi^{\prime}(t)\right) d t \\
& \leq \xi(a) \psi(a)+\int_{0}^{a} \frac{\xi(a)}{a} t\left(-\psi^{\prime}(t)\right) d t
\end{aligned}
$$


where we also used the monotonicity of both $\xi(t) / t$ and $\psi(t)$. We now further integrate by parts the last integral, so to get

$$
\int_{0}^{a} \xi^{\prime}(t) \psi(t) d t \leq \xi(a) \psi(a)-\frac{\xi(a)}{a} a \psi(a)+\frac{\xi(a)}{a} \int_{0}^{a} \psi(t) d t .
$$

This concludes the proof.

Lemma A.2. Let $N \geq 2$ and let $1 \leq q<2^{*}$, for every $L>0$ we set

$$
\Omega_{L}=\left(-\frac{L}{2}, \frac{L}{2}\right)^{N-1} \times(0,1) .
$$

Then we have:

(1) for $1 \leq q \leq 2$

$$
\lim _{L \rightarrow+\infty} L^{(N-1) \frac{2-q}{q}} \lambda_{2, q}\left(\Omega_{L}\right)=\left(\pi_{2, q}\right)^{2}, \quad \text { as } L \rightarrow+\infty .
$$

(2) for $2<q<2^{*}$

$$
\lim _{L \rightarrow+\infty} \lambda_{2, q}\left(\Omega_{L}\right)=\lambda_{2, q}\left(\mathbb{R}^{N-1} \times(0,1)\right)>0 .
$$

Proof. We distinguish again the two cases.

Case $1 \leq q \leq 2$. For $q=2$ this is contained for example in [5, Lemma A.2], we thus focus on the case $q<2$. By [5, equation (3.6)], we have

$$
\lim _{L \rightarrow+\infty} \lambda_{2, q}\left(\Omega_{L}\right)\left(\frac{\left|\Omega_{L}\right|^{\frac{1}{2}+\frac{1}{q}}}{P\left(\Omega_{L}\right)}\right)^{2}=\left(\frac{\pi_{2, q}}{2}\right)^{2},
$$

where $P\left(\Omega_{L}\right)$ stands for the perimeter of $\Omega_{L}$. If we now use that

$$
\left|\Omega_{L}\right|=L^{N-1} \quad \text { and } \quad P\left(\Omega_{L}\right) \sim 2 L^{N-1}, \quad \text { as } L \rightarrow+\infty,
$$

we get the desired result.

Case $2<q<2^{*}$. By monotonicity of $\lambda_{2, q}$ with respect to set inclusion, we have that

$$
\lambda_{2, q}\left(\Omega_{L}\right) \geq \lambda_{2, q}\left(\mathbb{R}^{N-1} \times(0,1)\right) \quad \text { and } \quad L \mapsto \lambda_{2, q}\left(\Omega_{L}\right) \text { is monotone decreasing. }
$$

Thus we get that

$$
\lim _{L \rightarrow+\infty} \lambda_{2, q}\left(\Omega_{L}\right) \geq \lambda_{2, q}\left(\mathbb{R}^{N-1} \times(0,1)\right)
$$

On the other hand, for every $\varepsilon>0$ we can take $\varphi_{\varepsilon} \in C_{0}^{\infty}\left(\mathbb{R}^{N-1} \times(0,1)\right)$ such that

$$
\lambda_{2, q}\left(\mathbb{R}^{N-1} \times(0,1)\right)+\varepsilon \geq \frac{\int_{\mathbb{R}^{N-1} \times(0,1)}\left|\nabla \varphi_{\varepsilon}\right|^{2} d x}{\left(\int_{\mathbb{R}^{N-1} \times(0,1)}\left|\varphi_{\varepsilon}\right|^{q} d x\right)^{\frac{2}{q}} .}
$$

Since $\varphi_{\varepsilon}$ has compact support, for $L$ large enough we get that $\varphi_{\varepsilon} \in C_{0}^{\infty}\left(\Omega_{L}\right)$, as well. This shows that for every $\varepsilon>0$

$$
\lambda_{2, q}\left(\mathbb{R}^{N-1} \times(0,1)\right)+\varepsilon \geq \lim _{L \rightarrow+\infty} \lambda_{2, q}\left(\Omega_{L}\right),
$$

and thus the claimed convergence of $\lambda_{2, q}\left(\Omega_{L}\right)$ follows. 
We are only left with showing that $\mathbb{R}^{N-1} \times(0,1)$ has a non-trivial Poincaré-Sobolev constant $\lambda_{2, q}$. By recalling that for a open convex set $\Omega \subset \mathbb{R}^{N}$ we have (see [6, Proposition 6.3])

$$
\lambda_{2, q}(\Omega) \geq \frac{C_{N, q}}{R_{\Omega}^{2+\frac{2-q}{q} N}},
$$

we get the desired assertion.

\section{REFERENCES}

[1] M. van den Berg, Estimates for the torsion function and Sobolev constants, Potential Anal., 36 (2012), 607-616. 2

[2] M. van den Berg, G. Buttazzo, A. Pratelli, On the relations between principal eigenvalue and torsional rigidity, preprint (2019), available at https://arxiv.org/abs/1910.14593 17

[3] M. van den Berg, V. Ferone, C. Nitsch, C. Trombetti, On a Pólya functional for rhombi, isosceles triangles, and thinning convex sets, preprint (2018), available at https://arxiv.org/abs/1811.04503 17

[4] M. van den Berg, V. Ferone, C. Nitsch, C. Trombetti, On Pólya's inequality for torsional rigidity and first Dirichlet eigenvalue, Integral Equations Operator Theory, 86 (2016), 579-600. 16, 17

[5] L. Brasco, On principal frequencies and isoperimetric ratios in convex sets, to appear on Ann. Fac. Sci. Toulouse Math., available at http://cvgmt.sns.it/paper/3891/2, 9, 11, 21

[6] L. Brasco, On principal frequencies and inradius in convex sets, Bruno Pini Mathematical Analysis Seminar 2018, 78-101, Bruno Pini Math. Anal. Semin. 9, Univ. Bologna, Alma Mater Stud., Bologna, 2018. 3, 19, 22

[7] L. Brasco, B. Ruffini, Compact Sobolev embeddings and torsion functions, Ann. Inst. H. Poincaré Anal. Non Linéaire, 34 (2017), 817-843. 6

[8] D. Bucur, G. Buttazzo, Variational Methods in Shape Optimization Problems. Progress in Nonlinear Differential Equations and their Applications, 65. Birkhäuser Boston, Inc., Boston, MA, 2005. 2, 12

[9] G. Buttazzo, S. Guarino Lo Bianco, M. Marini, Sharp estimates for the anisotropic torsional rigidity and the principal frequency, J. Math. Anal. Appl., 457 (2018), 1153-1172. 5

[10] T. Carroll, J. Ratzkin, Interpolating between torsional rigidity and principal frequency, J. Math. Anal. Appl., 379 (2011), 818-826. 2

[11] F. Della Pietra, N. Gavitone, S. Guarino Lo Bianco, On functionals involving the torsional rigidity related to some classes of nonlinear operators, J. Differential Equations, 265 (2018), 6424-6442. 5

[12] G. Franzina, P. D. Lamberti, Existence and uniqueness for a $p$-Laplacian nonlinear eigenvalue problem, Electron. J. Differential Equations, 26 (2010), pp. 1-10. 9

[13] A. Henrot, Extremum problems for eigenvalues of elliptic operators. Frontiers in Mathematics. Birkhauser Verlag, Basel, 2006. 2

[14] J. Hersch, Sur la fréquence fondamentale d'une membrane vibrante: évaluations par défaut et principe de maximum, Z. Angew. Math. Phys., 11 (1960), 387-413. 3

[15] R. Kajikiya, A priori estimate for the first eigenvalue of the $p$-Laplacian, Differential Integral Equations, 28 (2015), 1011-1028. 5, 6, 11

[16] B. Kawohl, Symmetry results for functions yielding best constants in Sobolev-type inequalities, Discrete Contin. Dynam. Systems, 6 (2000), 683-690. 14

[17] E. Makai, On the principal frequency of a membrane and the torsional rigidity of a beam. In "Studies in Math. Analysis and Related Topics", Stanford Univ. Press, Stanford 1962, 227-231. 4

[18] G. Pólya, G. Szegö, Isoperimetric Inequalities in Mathematical Physics. Annals of Mathematics Studies, no. 27, Princeton University Press, Princeton, N. J., 1951 4, 5

[19] M. H. Protter, A lower bound for the fundamental frequency of a convex region, Proc. Amer. Math. Soc., 81 (1981), 65-70. 3 
(L. Brasco) Dipartimento di Matematica e Informatica

Università degli Studi di Ferrara

Via Machiavelli 35, 44121 Ferrara, Italy

E-mail address: lorenzo.brasco@unife.it

(D. Mazzoleni) Dipartimento di Matematica e Fisica N. Tartaglia

Università Cattolica del Sacro Cuore

Via Trieste 17, 25121 Brescia, Italy

E-mail address: dariocesare.mazzoleni@unicatt.it 